\title{
CREACIÓN DE VALOR EN LA EMPRESA: LA ESTRATEGIA CONTINUA
}

\author{
CREATING VALUE IN THE COMPANY: THE CONTINUING STRATEGY \\ Jesús Martín Orna Barillas* \\ Unidad de Posgrado de la Facultad de Ciencias Contables \\ Universidad Nacional Mayor de San Marcos-UNMSM / Lima-Perú \\ [Recepción: Diciembre de 2014 / Conformidad: Diciembre 2014]
}

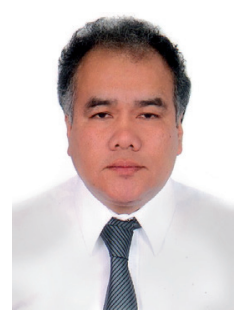

\section{RESUMEN}

En el entorno económico actual las empresas tienen que competir no solo contra otras empresas, sino también, por la creación de nuevos mercados que han de satisfacer las necesidades de los consumidores. Esto es dentro de su entorno externo. En el entorno interno tienen que modificar sus operaciones internas para el logro de la eficiencia y eficacia, lo que se traduce en la creación de valor para los consumidores mediante productos o servicios que satisfarán sus necesidades, crear una barrera de protección frente a la competencia, los trabajadores que serán recompensados por sus iniciativas en favor de la empresa, la empresa misma, mediante la mejora de sus procesos en base al logro de la eficiencia y reducción de costos con lo cual obtendrá una rentabilidad que le permitirá continuar con sus operaciones y para los propietarios que en base a la rentabilidad de la empresa, obtendrán beneficios económicos.

Lo cual nos indica que la empresa para que tenga rentabilidad sostenible en el tiempo, deberá generar valor internamente en sus procesos, analizando su cadena de valor, en concordancia con una adecuada planeación estratégica.

\section{Palabras clave:}

Creación de valor; cadena de valor; mercados; competencia; rentabilidad.

\begin{abstract}
In today's economic environment, companies have to compete not only against other companies, but also by creating new markets that meet the needs of consumers. This is within its external environment. In the domestic environment they have to change their internal operations for achieving efficiency and effectiveness, translated into the creation of value for consumers through products or services that meet their needs, it will create a protective barrier against competitors, workers will be rewarded for their efforts on behalf of the company, the company itself - by improving their processes based on the achievement of efficiency and cost reduction- will get a return that allows it to continue operations; and to owners, based on the profitability of the company, will gain economic benefits. Therefore it indicates that the company, to have sustainable profitability over time, must create value internally in their processes, analyzing the value chain, in line with a proper strategic planning.
\end{abstract}

\section{Keywords:}

Value creation; value chain; markets; competition; profitability.

\footnotetext{
* Maestrista en Contabilidad con Mención en Contabilidad de Gestión - UNMSM. Contador Público Colegiado - UNFV. Email: jesusob77777@yahoo.es
} 


\section{INTRODUCCIÓN}

La gestión empresarial en el siglo XXI comprende la adopción de políticas mediante la aplicación de estrategias, tácticas, procesos, técnicas y buenas prácticas para el cumplimiento de la visión y misión; así como, proveer información para la toma de decisiones para obtener una ventaja competitiva sobre la competencia. Dentro de la gestión se incluye la planeación, organización, dirección y control. Estas cuatro actividades permitirán a la empresa a mantenerse en el mercado, consolidarse y, posteriormente, expandirse.

\section{LA ESTRATEGIA}

Las empresas deben ser flexibles para responder rápidamente a los cambios de la competencia y el mercado. Deben compararse continuamente con las mejores para optimizar sus prácticas. (Porter, 2009a:69)

Para ello, tienen que evolucionar en su pensamiento organizacional y de dirección, lo cual involucra el control y medición de sus acciones en todas las áreas de las empresas para poder sostenerse, consolidarse en el mercado, expandirse y continuar tratando de lograr la mayor captación posible del mercado, que les proporcione una rentabilidad para la continuidad de sus operaciones; además, de conocer y reconocer las fortalezas y debilidades de sus competidores.

Estos requerimientos que se impone la gestión de las empresas, constituye y da forma a la estrategia.

Una estrategia diferente y superior adoptada por una empresa significa la creación de una ventaja competitiva, que le permita posicionarse en una gran parte del mercado.

Una ventaja competitiva genera una rentabilidad superior al de la competencia. Esta rentabilidad depende de tres factores: 1) el valor que los clientes asignan a los productos, 2) el precio que una empresa fija por sus productos y 3 ) los costos de crear tales productos. (Hill \& Jones, 2011a:77).

Estos tres factores se encuentran en tres niveles o áreas de la empresa: la financiera, la de clientes y la de operación o producción. Lo que constituye una cadena de actividades, que en la práctica se conoce como cadena de valor.

Las estrategias más conocidas y adoptadas por las empresas son las estrategias genéricas de Porter (Porter, 2009b:78) que son:
A. Liderazgo en Costos
B. Diferenciación
C. Segmentación

\section{LA CADENA DE VALOR}

Se entiende a la cadena de valor como la idea de que la empresa es un conjunto de actividades concatenadas entre sí, que transforma los insumos en productos que los clientes valoran y que satisfacen sus necesidades de consumo, originando una rentabilidad para la empresa y produciendo un beneficio para la misma y para los propietarios o accionistas, lo que significa la creación de valor en base a todo el proceso de las actividades de la empresa. (Hill \& Jones, 2011b:81).

Al utilizar la cadena de valor, la empresa obtiene:

a. Un esquema para poder determinar en qué área incidir para la creación de valor.

b.Una herramienta de gestión para definir las acciones para desarrollar una ventaja competitiva sostenible en el tiempo, lo cual implica elaborar una Planificación Estratégica para la creación de valor.

\section{LA PLANEACIÓN ESTRATÉGICA}

Drucker señala: "la planeación estratégica es el proceso continuo, basado en el conocimiento más amplio del futuro considerado, que se emplea para tomar decisiones en el presente, las cuales implican riesgos futuros en razón de los resultados esperados: es organizar las actividades necesarias para poner en práctica las decisiones y para medir, con una reevaluación sistemática, los resultados obtenidos frente a las expectativas que se hayan generado". (Chiavenato \& Sapiro, 2011:26). 
En el siguiente cuadro, del proceso básico de la planeación estratégica, la gerencia debe incidir en:

\section{Cuadro No 01:}

Planeación Estratégica: Ambientes

\begin{tabular}{|l|l|}
\hline \multicolumn{1}{|c|}{ Ambiente Interno } & \multicolumn{1}{c|}{ Ambiente Externo } \\
\hline Eliminar las ineficiencias dentro de la empresa. & Anticipar las tendencias del mercado. \\
\hline Construir la Cadena de Valor de la empresa. & Anticipar los cambios tributarios. \\
\hline $\begin{array}{l}\text { Analizar constantemente las fortalezas y debilida- } \\
\text { des de la empresa. }\end{array}$ & Adoptar los cambios tecnológicos necesarios. \\
\hline $\begin{array}{l}\text { Elaborar una información estratégica para que la } \\
\text { Gerencia tome las mejores decisiones. }\end{array}$ & $\begin{array}{l}\text { Tener una fuerte capacidad de respuesta frente a } \\
\text { las acciones de la competencia. }\end{array}$ \\
\hline
\end{tabular}

Fuente: Elaboración propia.

\section{CREACIÓN DE VALOR}

En el ámbito del management, la generación de valor es un tema de preocupación constante, de relevancia estratégica y de vigente actualidad para los estrategas empresariales, administradores de empresas y de cualquier tipo de organización, sea en marcha o en proceso de formación ( García, 2012:25).

Para tener una perspectiva nacional consideramos los resultados de la entrevista que se ha realizado a cuarenta (40) ejecutivos con influencia decisiva sobre el significado de la generación de valor. Los resultados se detallan a continuación:

a. La mayoría afirmó que el valor era referente a la satisfacción de los accionistas y/o dueños de la empresa; en segundo término, de los clientes y finalmente, del personal interno de la empresa.

b.Un grupo considerable también afirmó que la generación de valor se debe basar en una propuesta que diferencia a la empresa de la competencia.

c. Un pequeño grupo afirmó que el valor es más que la mera organización y que implicaba a la sociedad y a los stakeholders externos involucrados.

d. Se produjo un consenso entre los encuestados acerca de que la propuesta de valor de una empresa, es un tema fundamental para su desarrollo y éxito empresarial.
De este resultado se concluye en primer lugar, que la mayoría de los ejecutivos focalizan la generación de valor en los accionistas y los clientes en primer lugar; luego incluyen a los trabajadores.

Considerando lo anterior, nosotros planteamos que una empresa debe afrontar cinco (05) problemas fundamentales para la creación de valor: el valor esperado que esperan los propietarios o accionistas por su inversión; el valor esperado por los clientes; la creación de valor expresado en la satisfacción del personal interno de la empresa; la creación de valor para hacer frente a la competencia (protección) y la creación de valor para la misma empresa.

\section{1.- CReación de VAlor Para la EMPRESA}

El análisis de los recursos y capacidades determinan los factores para poder competir competitivamente en el mercado, con lo cual se creará valor.

Un recurso es un insumo productivo o un activo competitivo que una empresa tiene, como por ejemplo una marca o una maquinaria que produce en el menor tiempo posible un determinado producto. (Thompson y otros, 2012:95).

Analizando los recursos de una empresa, los podemos dividir en tangibles e intangibles que se detallan en el siguiente cuadro: 


\section{Cuadro No 02:}

Recursos de una empresa

\begin{tabular}{|c|c|}
\hline Recursos Tangibles & Recursos Intangibles \\
\hline $\begin{array}{l}\text { a. Físicos: Derechos de propiedad, predios, ubica- } \\
\text { ciones de tiendas, canales de distribución. } \\
\text { b. Financieros: Efectivo, valores negociables, capa- } \\
\text { cidad de endeudamiento y capacidad crediticia. } \\
\text { c. Tecnológicos: Patentes, derechos de autor, pro- } \\
\text { cesos tecnológicos, etc. } \\
\text { d. Organizacionales: Sistemas de Tecnología de la } \\
\text { Información y Comunicaciones (TIC`s). }\end{array}$ & $\begin{array}{l}\text { a. Capital Intelectual: Experiencia, aprendizaje y co- } \\
\text { nocimientos del personal para los procesos or- } \\
\text { ganizacionales, como reducir costos operativos, } \\
\text { mejorar la calidad del producto, innovación y de- } \\
\text { sarrollo de mejores productos y servicios. } \\
\text { b. Marcas, imagen de la empresa, lealtad del cliente, } \\
\text { reputación en cuanto a la calidad del producto o } \\
\text { servicio, reputación con respecto a los proveedo- } \\
\text { res y en lo concerniente a la Responsabilidad So- } \\
\text { cial Empresarial. }\end{array}$ \\
\hline
\end{tabular}

Fuente: Elaboración propia.

Una capacidad es la habilidad de una empresa de desempeñar una actividad de manera más eficiente que el promedio de la competencia.

La identificación de las capacidades es un proceso más complejo aún. Éstas se forman a partir de los recursos. Es en base a ellos con los cuales se puede dirigir a la empresa. En el siguiente cuadro detallamos las principales capacidades.

\section{Cuadro No 03:}

Capacidades de la empresa

a. Sòlida posición financiera.

b. Fuerte presencia de marca en el mercado.

c. Gran base de clientes.

d. Lealtad de los clientes.

e. Sólida posición para negociar con compradores y proveedores.

f. Contar con una excelente cadena de suministro.

g. Calidad superior del producto.

h. Excelentes canales de distribución.

i. Gran cobertura geográfica de los canales de distribución.

j. Costo y precio competitivo.

k. Tendencia a expandirse a nuevos mercados.

1. Maquinaria de producción superior a la competencia.

m. Personal competitivo.

n. Utilización al máximo de la TIC`s.

Fuente: Elaboración propia.

\section{Creación de valor frente a la COMPETENCIA (PROTECCIÓN)}

Para que la empresa sea lo suficientemente competitiva para enfrentar a la competencia, debe de realizar dos acciones fundamentales:

a. Determinar si los recursos y capacidades son lo suficientemente fuertes para enfrentar a la competencia.

Para ello, se deben formular las siguientes preguntas:

1. ¿Son competitivos los recursos y capacidades?

2. ¿La competencia carece de algunos o varios de nuestros recursos o capacidades?

3. ¿La competencia tiene similares recursos o capacidades de la empresa?

b. Identificar a la competencia.

Este proceso es un factor clave para poder determinar en qué situación la empresa se encuentra en referencia frente a la competencia. Se deberá realizar un análisis y evaluación de los competidores en los aspectos de estructura, financiero, de producción, de mercados, instalaciones, recursos humanos y sus estrategias corporativas y de marketing.

\section{Creación de valor para los trabajadores}

Si entendemos por personas con talento, aquellas que contribuyen a los resultados de la organización, sea individual o colectivamente, queda patente que existe un vínculo muy estrecho con la estrategia. 
En este contexto, muchas empresas se centran en las actividades que mejor controlan o saben hacer, con la consecuencia de valorar más a los empleados con los conocimientos y habilidades críticos del negocio. El auge del conocimiento y el impacto de la innovación hacen que gestionar el talento de manera eficaz implique crear valor a la empresa.

En vista de que los trabajadores con talento logran crear valor para la empresa, esta les retribuye de la mejor forma posible para retenerlos, asegurar de esta forma la continua creación de valor. Para ello, deberá establecer una política de retención del talento, lo cual origina una mutua reciprocidad de creación de valor.

\section{Creación de valor para los Clientes}

Habiendo determinado si la empresa es lo suficientemente competitiva para enfrentar a la competencia, se tiene otro punto determinante en la vida económica de la empresa. El valor que los potenciales clientes deben de obtener por la compra de los productos o servicios que la empresa ofrece. Lo cual se ha de mostrar en el siguiente diagrama:

\section{Diagrama $\mathbf{N}^{\circ} 01$ :}

Creación de valor para los clientes

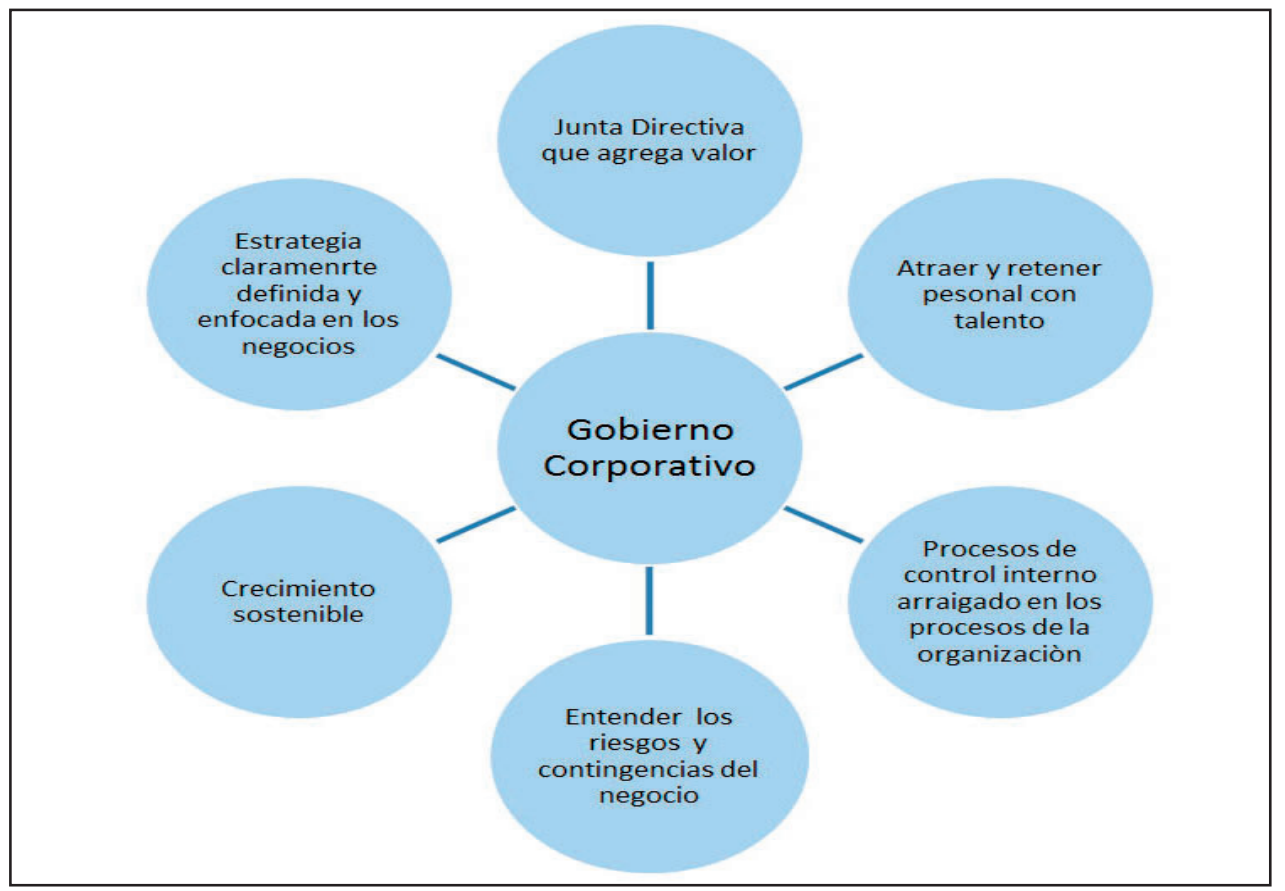

Fuente: Elaboración propia.

Asimismo, es necesario que tambien la empresa, tenga en cuenta los factores sociales, económicos y tecnológicos que tan velozmente cambian en la actualidad, puesto que la lucha es tan intensa por la conquista de nuevos segmentos de mercado que no se puede obviar los siguientes factores para la obtención de clientes:

1. Innovación tecnológica.

2. Investigación y desarrollo.

3. Globalización.

4. Mayor poder de compra de los clientes.
5. Intensa competencia.

6. Canales de distribución.

7. Venta directa.

8. Comercio electrónico

9. Asesoramiento al cliente.

10. Compromiso con el cliente.

11. Procesos internos.

12. Costos.

13. Diseño del producto o servicio.

14. Servicio de post venta.

15. Aspectos tributarios. 


\section{Creación de valor para los accionistas O PROPIETARIOS}

Los accionistas o dueños de la empresa reciben el valor cuando tienen una empresa sana y sólida que crece y genera rentabilidad. La esencia del espíritu empresarial se basa en la idea de crear una empresa para generar rentabilidad.

La utilización de las alternativas financieras y económicas de acuerdo a las decisiones de gestión de los activos y pasivos de la empresa deberá de determinar una posible rentabilidad o pérdida para los accionistas o propietarios de la empresa. Lo cual ha de originar la creación o destrucción de valor en la empresa, que se determinará mediante un análisis y diagnóstico integral.

Para realizar un análisis y diagnóstico integral de la empresa, no bastará tomar como referencia los indicadores financieros tradicionales, se deberá tomar indicadores que sean cuantitativos y cualitativos en sus características y que contribuyan a la obtención de una rentabilidad y diferenciación de la empresa frente a la competencia. Estos últimos son de la clase que se consideran intangibles.
Nosotros consideramos a los intangibles como los factores que carecen de forma corpórea y de solidez. Aplicados a la gestión se relacionan con el prestigio de la marca, la calidad de los procesos internos de producción, la fidelidad de los clientes, la especialización de los empleados, etcétera.

"Las medidas financieras son inadecuadas para guiar y evaluar las trayectorias de la organización a través de los entornos competitivos. Les faltan indicadores que reflejen gran parte del valor que ha sido creado o destruido por las acciones de los directivos durante el período contable más reciente. Los indicadores fin ancieros nos dicen algo pero no todo, sobre la historia de las acciones pasadas, y no consiguen proporcionar una guía adecuada para las acciones que hay que realizar hoy y el día después para crear un valor financiero futuro" (Kaplan \& Norton, 2011:43-44).

Por lo tanto, es necesario analizar e indicar los posibles errores que se cometen al no tener indicadores no financieros que proporcionarán información de la situación real en que se encuentra la empresa. Estos errores podríamos clasificarlos en torno a los clientes, a los procesos internos y a los trabajadores, tal como se aprecia en el siguiente cuadro:

\section{Cuadro No 04:}

Posibles errores al no tener indicadores no financieros

\begin{tabular}{|l|l|l|}
\hline \multicolumn{1}{|c|}{ Clientes } & \multicolumn{1}{|c|}{ Procesos Internos } & \multicolumn{1}{c|}{ Trabajadores } \\
\hline Insatisfacción de los clientes. & Productos y/o servicios con defectos. & $\begin{array}{l}\text { Poco especializados en la reparación } \\
\text { de los productos o en la prestación de } \\
\text { servicios. }\end{array}$ \\
\hline $\begin{array}{l}\text { Falta de respuesta rápida para la } \\
\text { atención de los clientes. }\end{array}$ & $\begin{array}{l}\text { Falta de innovación y desarrollo de } \\
\text { productos y/o servicios mejores. }\end{array}$ & $\begin{array}{l}\text { Exceso de trabajadores en el área de } \\
\text { ventas y falta de ellos en el área de de- } \\
\text { sarrollo de producción de los produc- } \\
\text { tos y/o servicios. }\end{array}$ \\
\hline $\begin{array}{l}\text { Carencia de programa de fideli- } \\
\text { zación del cliente. }\end{array}$ & $\begin{array}{l}\text { Falta de control de calidad de la pro- } \\
\text { ducción de los bienes y/o servicios. }\end{array}$ & $\begin{array}{l}\text { Falta de visión a largo plazo de los eje- } \\
\text { cutivos. }\end{array}$ \\
\hline $\begin{array}{l}\text { Falta de una adecuada evalua- } \\
\text { ción de las necesidades de los } \\
\text { clientes. }\end{array}$ & $\begin{array}{l}\text { Olvido del giro principal de la empre- } \\
\text { sa. }\end{array}$ & $\begin{array}{l}\text { Carencia de liderazgo para analizar la } \\
\text { empresa frente a la competencia. }\end{array}$ \\
\hline $\begin{array}{l}\text { Falta de creación de valor para } \\
\text { el cliente reduciendo sus costos, } \\
\text { desconociendo el potencial de la } \\
\text { competencia. }\end{array}$ & $\begin{array}{l}\text { Repetición de los errores en la pro- } \\
\text { ducción olvidando la creación de valor } \\
\text { servicios para la empresa. }\end{array}$ & $\begin{array}{l}\text { Falta de compromiso con el cliente en } \\
\text { solucionar sus problemas. }\end{array}$ \\
\hline
\end{tabular}

Fuente: Elaboración propia. 


\section{CONCLUSIONES}

1. La empresa deberá crear valor en forma continua, caso contrario será desplazada por sus competidores.

2. La gestión de la empresa que solo considera generar valor para sus propietarios, está en un error.

3. En la actualidad la empresa deberá generar valor para la misma empresa, para su protección, trabajadores, clientes y propietarios.

4. Para determinar la creación de valor positivo o negativo, se deberá tener en consideración indicadores intangibles aparte de los tradicionales financieros clásicos.

5. Las empresas al tener una planeación estratégica tendrán un desempeño superior a las que no la tienen, posibilitando la creación de valor. Las que tienen éxito han de procurar que las estrategias a utilizar se adapten al entorno externo sin olvidar el interno, el cual ha de especificar los procesos internos de la empresa.

6. La planeación estratégica, es considerada como un proceso integral de la empresa que se inicia con la determinación clara de la visión y misión para poder lograr los objetivos establecidos.

\section{RECOMENDACIONES}

1. Las empresas deberán establecer como meta básica la creación de valor no solo para los propietarios; sino también, deberán considerar a los trabajadores, clientes, a la empresa misma y establecer una creación de valor como protección para hacer frente a la competencia.

2. Considerar en el diagnóstico de la creación de valor a indicadores intangibles para realizar una evaluación integral de la empresa.
3. Implementar periódicamente una planeación estratégica.

4. Evaluar continuamente la cadena de valor.

5. Revisar continuamente las estrategias para una posible retroalimentación o adopción de otras.

\section{REFERENCIAS BIBLIOGRÁFICAS}

1. CHIAVENATO, I. \& Sapiro, A. (2011). Planeación Estratégica, fundamentos y aplicaciones. Segunda edición. México: McGraw-Hill/Interamericana Editores, S.A.

2. Escuela de Postgrado de la UPC. Cuaderno de Investigación EPG. Edición No. 15 - Julio 2011. Lima.

3. GARCÍA, E. (2012). ¿Cómo Generar valor en las empresas? Lima: Universidad del Pacífico.

4. HILL, Ch. \& JONES, G. (2011). Administración Estratégica, un enfoque integral. Novena edición. México: Cencage Learning.

5. KAPLAN, R. \& NORTON, D. (2011). El Cuadro de Mando Integral. Tercera Reimpresión. Barcelona: Gestión 2000. Grupo Planeta.

6. PORTER, Michael E. (2009) Estrategia Competitiva. Madrid: Ediciones Pirámide Grupo Anaya S.A.).

7. PORTER, Michael E. (2009) Ser Competitivo. Barcelona: Ediciones Deusto.

8. RAMÍREZ, D. (2013). Contabilidad Administrativa. Novena edición. México: McGraw-Hill/ Interamericana Editores, S.A. DE C.V.

9. THOMPSON, A., PETERAF. M., GAMBLE, J. \& STRICKLAND. A. (2012). Administración Estratégica, Teoría y Casos. Décimo Octava Edición. México: McGraw-Hill/Interamericana Editores, S.A. 